East African Medical Journal Vol. 83 No. 12 December 2006

MANAGEMENT OF TRANSITIONAL CELL CARCINOMA OF THE URINARY BLADDER AT KENYATTA NATIONAL HOSPITAL, NAIROBI

C.G. Waihenya, MBChB, MMed (Surg.), Urology (Stell. South Africa), Thika District Hospital, P.O. Box 6117, Thika, Kenya and P.N. Mungai, MBChB, MMed (Surg.), Urology, RCS (England), Senior Lecturer, Department of Surgery, College of Health Sciences, University of Nairobi, P.O. Box 19676, Nairobi, Kenya

\title{
MANAGEMENT OF TRANSITIONAL CELL CARCINOMA OF THE URINARY BLADDER AT KENYATTA NATIONAL HOSPITAL, NAIROBI
}

\author{
C.G. WAIHENYA and P.N. MUNGAI
}

\begin{abstract}
Objectives: To highlight the management of patients with transitional cell carcinoma of the urinary bladder with regards to clinical presentation, treatment and outcome.

Design: A retrospective study.

Setting: Kenyatta National Hospital, Nairobi, Kenya.

Subjects: Fifty two patients who presented at Kenyatta National Hospital over a ten year period with histologically proven transitional cell carcinoma of the urinary bladder. There were 41 males and 11 females aged 27 to 84 years. The mean age was 57 years.

Results: An average of 5.2 patients per year were seen. The male to female ratio was 3.7:1. Most common clinical presentations were haematuria $98 \%$, Lower abdominal pains $71 \%$. The main investigative procedures done in these patients included cystoscopy in $71.2 \%$, ultrasound $46.2 \%$, IVU $32.7 \%$ among others. The treatment modalities were based on the stage of the disease and included surgery $48.1 \%$, combination therapy in $23.1 \%$, chemotherapy in $5.8 \%$ and radiotherapy in $3.8 \%$. Surgery was the mainstay of treatment, cystectomy was done in $26.9 \%$ and cystostomy and resection of tumour $26.9 \%$. Other surgical methods carried out were transurethral resection of bladder tumour(TURBT), cystectomy and bladder augumentation, channel transurethral resection, cystectomy and ileocondult. Nine patients $(17.3 \%)$ were not given any treatment because either the disease was too advanced and died before any treatment was instituted or were lost to follow up. Mortality and outcome of the disease was difficult to assess due to poor follow up, however $65.4 \%$ of the patients were still alive, $17.3 \%$ had died and $17.3 \%$ were lost to follow up by the end of the study period.

Conclusion: Haematuria was the most important presenting clinical feature. Poor record keeping may have contributed to the low number of patients enrolled into the study. The TCC in this study was not thoroughly managed. It is suggested that early diagnosis, early surgery and combination of other treatment modalities should improve the outcome. This can only be possible with further training of health personnel, the education of the public and availability of improved diagnostic as well as treatment facilities especially cystoscopes and resectoscopes. There is need for developing proper management protocols for bladder tumours.
\end{abstract}

\section{INTRODUCTION}

The most common presenting symptom of transitional cell carcinoma of the urinary bladder is painless haematuria, which occurs in about $85 \%$ of patients (1). The symptom complex of bladder irritability with urinary frequency, urgency, and dysuria is the second most common form of 
presentation and usually is associated with diffuse carcinoma in situ or invasive bladder cancer. Other signs and symptoms include flank pain from ureteral obstruction, lower extremity oedema, and a pelvic mass. Occasionally, patients present with symptoms of advanced disease such as weight loss and abdominal or bone pain.

Currently, urine cytology remains the standard non-invasive in vitro test to detect primary and recurrent disease. Blood group antigens $A B O$ and lewis $X$, bladder tumour antigen (BTA) test (Bard Urological Covington, USA) and nuclear matrix protein 22 (NMP22), Flow cytometry and Quantitative flourescent image analysis are useful techniques in use (2-6).

Cystoscopy and biopsy: All patients require a cystoscopy, and the pathologist must confirm the diagnosis, not by urological opinion. All abnormal areas of bladder mucosa should be biopsied. The findings of carcinoma in situ or even severe dysplasia may adversely affect the prognosis. Tumours are resected primarily to establish pathological type, grade and stage. The presence of muscle invasion in a biopsy specimen means that other imaging modalities will be required usually $\mathrm{CT}$ of the pelvis and abdomen. Treatment depends on the stage of disease. Patients presenting with favourable prognostic factors (solitary well differentiated, early stage small tumours) are often treated adequately by transurethral resection (TUR) alone. The addition of one dose of an intravesical chemotherapeutic agent has been shown to reduce the recurrence rate, but only further long term follow-up will demonstrate conclusively whether the progression rate in these patients (which may be less than $2 \%$ ) has also been reduced (7). Patients presenting with multiple tumours, or who have recurrence at first cystoscopy, have a significantly higher rate of subsequent recurrence, and therefore a potential for progression.

There is a controversy about the type of intravesical agent that should be used. In recent studies, all agents tested appear to reduce the recurrence rate, it remains to be determined in suitably size multicentric studies whether reductions in recurrence rate are associated with reductions in progression rate. Some studies appear to show a definite benefit of BCG immunotherapy over intravesical chemotherapy with adriamycin, however, studies from Europe show no difference between BCG and mitomycin $C$ in the reduction of recurrence rate $(8,9)$. Intravesical instillation of INF $\alpha$ $2 \mathrm{~b}$ has also been used in reducing recurrence rate in superficial transitional cell carcinoma. Upto $50 \%$ of patients with carcinoma in situ may progress rapidly to invasive disease. The role of the newer intravesical therapy e.g. (megadose vitamins, $\alpha$-interferon) are still undergoing demonstration in randomised prospective studies.

On surgery as a mode of management world wide, most patients presenting with muscle invasive transitional cell carcinoma are treated by radical cystectomy with formation of a conduit drainage system or orthotopic bladder substitution. Radiotherapy is done in patients unfit for surgery or unwilling to accept consequences of surgery. These patients tend to be older and more frail, and possibly have a more advanced disease than a comparable cystectomy group.

The use of chemotherapy, as either neo-adjuvant or adjuvant treatment along - side definitive therapy, has often resulted in five-year survival rates of 60$70 \%$ in small series. Transitional cell carcinoma is sensitive to cisplatin. Neoadjuvant chemotherapy has shown no definitive improvement in survival compared with modern series of cystectomy alone and a low rate $(20 \%)$ of disease free bladder preservation $(10,11)$. Chemotherapy combination using M-VAC (methotrexate, vinblastine, adriamycin and cisplatin) and CMV (cisplatin, methotrexate, and vinblastine) appear to show extremely good response rates that are durable and accompanied by disease free interval. Paclitaxel, docetaxel and gemcitabine are recent drugs in the market.

Follow-up cystoscopy and urography have a role in management of TCC. The traditional followup programme recommended for patients with superficial bladder cancer includes serial cystoscopies every three months for two years, then every six months for two years and then yearly. Either annual or biennial excretory urograms have been recommended. Other investigators believe that patients should be followed with cytology examination and cystoscopy less frequently.

\section{MATERIALS AND METHODS}

The study was carried out at Kenyatta National Hospital (KNH). This was a retrospective study 
covering a period of ten years from January 1990 to December 1999. Only those patients with histologically confirmed diagnosis of transitional cell carcinoma of the urinary bladder treated at $\mathrm{KNH}$ during the study period were included. Patients with incomplete medical records, unavailable histology results were excluded from the study. The sample size was determined by the study period. The relevant records of the patients with TCC were reviewed after approval of the study proposal by the KNH Research Committee. Data were collected using tally sheets and analysed using statistical computer programme (SPSS).

\section{RESULTS}

Two hundred and twenty four patients were clinically diagnosed to have urinary bladder cancer. Of these 127 (57\%) files were traceable from which 79 had histologically proven bladder cancer. Fifty two patients of these were transitional cell carcinoma and were enrolled into the study.

Clinical presentation: Patients presented with various signs and symptoms, namely haematuria 51 patients (98.1\%), low abdominal pains 37 patients (71.2\%), pelvic mass 19 patients (36.5\%), dysuria 17 patients $(32.7 \%)$. Haematuria was the most common presenting symptom. Most of the signs and symptoms occurred in combination (Table 1).

Investigative procedures: Various investigations were carried out on the patients. Most of them, $37(71.2 \%)$ had cystoscopy and biopsy done. Others were urine cytology in seven patients (13.5\%), open cystostomy and biopsy done in other centres before patients were referred to Kenyatta National Hospital (Table 2). Radiological investigation included Ultrasonography in 24 patients (46.2\%), CT scan in three patients $(5.8 \%)$, IVU in 17 patients $(32.7 \%)$, plain abdominal X-ray in one patient (1.9\%). None of the patients had MRI as an investigative procedure. The most common stage of TCC was muscle invasive in 22 patients (42.3\%), superficial in 20 patients $(38.5 \%)$, metastatic nine patients (17.3\%) and carcinoma in situ one patient (1.9\%) (Table 2). Overall majority of the patients had invasive disease $59.6 \%$ as compared to superficial $40.4 \%$.
Management: Various modes of management of transitional cell carcinoma were used for the 52 patients seen at the hospital over the study period. The modalities included surgical procedures, radiotherapy, chemotherapy and a combination of any two. The most common modality of management was surgery $48.1 \%$, combination therapy in $23.1 \%$ of the patients. Chemotherapy accounts for $5.8 \%$ of the patients and radiotherapy $3.8 \%$ of the patients. Various chemotherapeautic agents were used. mainly in combination, they included vincristine (V), cyclophosphamide (C), doxorubicin (adriamycin-A), methotrexate (M), cisplatin (C). Combination MVAC was used in one patient after recurrence following TURBT. Systematic vincristine and adriamycin were given to one other patient. Intravesical doxorubicin was used in several patients. Combination therapy was given to 12 patients (23.1\%). These patients received either surgery and radiotherapy in seven patients, or surgery and chemotherapy in five patients.

Surgical management: Various surgical procedures were carried out on the patients of transitional cell carcinoma seen over ten years period. Table 4 summarises the procedures. The most common type of surgical procedure carried out was partial cystectomy in 14 patients (26.9\%) and cystostomy and excision in 14 patients $(26.9 \%)$. Others were transurethral resection in eight patients $(15.4 \%)$, cystectomy and ileoconduit in three patients $(5.8 \%)$, cystectomy and bladder substitution in two patients $(3.8 \%)$, channel transurethral resection to relieve urinary obstruction in one patient $(1.9 \%)$, and cystectomy and ureterosigmoidostomy were done in one patient $(1.9 \%)$.

Outcome and follow-up of patients: Within the ten year period of study $67.4 \%$ of the patients were lost to follow-up, $7.6 \%$ of the patients died within one year after diagnosis and another $25 \%$ were alive two years later (Table 5). The two patients who died after six months had more advanced disease compared to those who died after one year. One had metastasis to the liver and the uterus while the other had mets to the regional nodes. Both had poorly differentiated tumour. For those who died within one year both had poorly differentiated muscle invasive disease but had no metastasis. One died of severe haematuria. 
Table 1

Gender characteristics and clinical features

\begin{tabular}{lcc}
\hline Characteristic & No. & $(\%)$ \\
\hline Signs/Symptoms & & \\
$\quad$ Haematuria & 51 & 98.1 \\
$\quad$ Lower abdominal pains & 37 & 71.2 \\
$\quad$ Pelvic mass & 19 & 36.5 \\
$\quad$ Dysuria & 17 & 31.7 \\
Gender characteristics & & \\
$\quad$ Sex & & \\
Male & 41 & 78.8 \\
$\quad$ Female & 11 & 21.1 \\
\hline Total & 52 & 100 \\
\hline
\end{tabular}

Table 2

Investigative procedures

\begin{tabular}{lcc}
\hline Investigation & No. & $(\%)$ \\
\hline Cystoscopy & 37 & 71.2 \\
Ultrasound & 24 & 46.2 \\
IVU & 17 & 32.7 \\
Urine cytology & 7 & 13.5 \\
Open cystostomy & 5 & 9.6 \\
CT scan & 3 & 5.8 \\
KUB & 1 & 1.9 \\
MRI & 0 & 0 \\
\hline
\end{tabular}

Table 3

Management

\begin{tabular}{lcc}
\hline Mode & No. & $(\%)$ \\
\hline Surgical & 25 & 48.1 \\
Combination & 12 & 23.1 \\
Palliation & 10 & 19.2 \\
Chemotherapy & 3 & 5.8 \\
Radiotherapy & 2 & 3.8 \\
\hline Total & 78 & 100 \\
\hline
\end{tabular}

Table 4

Surgical management

\begin{tabular}{lcc}
\hline Surgical Procedure & No. & $(\%)$ \\
\hline Partial cystectomy & 14 & 26.9 \\
Cystostomy and excision & 14 & 26.9 \\
TURBT & 8 & 15.4 \\
Cystectomy and ileoconduit & 3 & 5.8 \\
Cystectomy and bladder substitution & 2 & 3.8 \\
Cystectomy and ureterosigmoidostomy & 1 & 1.9 \\
Channel TUR & 1 & 1.9 \\
None & 9 & 17.5 \\
\hline Total & 78 & 100 \\
\hline
\end{tabular}

Table 5

Outcome and follow-up of patients

\begin{tabular}{lcc}
\hline Outcome & No. & $(\%)$ \\
\hline Died within six months & 2 & 3.8 \\
Died within one year & 2 & 3.8 \\
Alive after two years & 13 & 25.0 \\
Lost to follow-up & 35 & 67.4 \\
\hline Total & 78 & 100 \\
\hline
\end{tabular}

\section{DISCUSSION}

In this study out of the 52 patients, haematuria was found in 51 patients (98.1\%), lower abdominal pains in 37 patients $(71.2 \%)$, pelvic mass in 19 patients $(36.5 \%)$ and dysuria in 17 patients (32.7\%). Pelvic mass was a sign of advanced disease.

Presentation of transitional cell carcinoma depends on the clinical stage of the disease, presence or absence of metastases, haematuria being the most common presentation (12).

The investigations were done in various combinations. The most common investigative procedures for diagnostic purpose in this study were cystoscopy performed in 37 patients (71.2\%), ultrasonography in 24 patients (4:6.2\%), IVU in 17 patients $(32.7 \%)$, urine cytology in seven patients $(13.5 \%)$, plain abdominal $X$-rays in one patient (1.9\%). Open cystostomy and biopsy was done in five patients $(9.6 \%)$ in other centres before admission to Kenyatta National Hospital. Cystostomy and biopsy were done in other health centres before referral. This is only mentioned to be condemned for it encourages seeding and spread of tumour cells $(13,14)$. Urine cytology was not a favoured mode of investigation for it is not a cost effective means of screening for bladder cancer.

Of the various stages of transitional cell carcinoma muscle, invasive was the most common accounting for 22 patients $(42.3 \%)$ followed by superficial in 20 patients $(38.5 \%)$ metastatic in nine patients (17.3\%) and ca in situ in one patient (1.9\%). Many early stage tumours could have been missed and many patients presented late (6). Muscle invasive histological stage accounts for the majority of patients of transitional cell carcinoma across the age spectrum.

Management of these tumours calls for a multidisciplinary approach. Definitive management options depend on the stage and grade of the disease. For superficial tumours (early stage tumours Cis,Ta,T1) the main stay of treatment is TUR alone. In this study the most common mode of management employed was surgery in 25 patients $(48.1 \%)$, combination in 12 patients $(23.1 \%)$, chemotherapy in three patients $(5.8 \%)$ and radiotherapy in two patients $(3.8 \%)$. Ten patients $(19.2 \%)$ received no treatment, they either did not comply, died before treatment or were lost to follow-up.

Immunotherapy with intravesical BCG, Interferons or megadose vitamins were not used on 
any of the patients. On the outcome, mortality and follow up of patients, the data on these were not found to be useful because of the fact that the followup of these patients was poor. Within the ten-year period of study $67.4 \%$ of the patients were lost to follow-up, $7.6 \%$ died within one year after diagnosis and another $25 \%$ were alive two years later. Those who died within six months had distant metastasis while those who lived to one year had muscle invasive disease without distant metastasis. It is likely that the cost of traveling back to Nairobi may have hindered patients from coming back for follow up. Others may have died at home or the nearest health institution before coming back for their next appointment. This is despite the fact that they were discharged with instructions to have follow-up in both the radiotherapy and urology outpatient clinic. Some patients were lost to follow-up when upon discharge from hospital were referred to the radiotherapy clinic. Follow-up cystoscopy can be readily performed as an office procedure by use of flexible or rigid cystoscopies( 15).

In conclusion, haematuria was the most important presenting clinical feature. Poor record keeping may have contributed to the low number of patients enrolled into the study. The TCC in this study was not thoroughly managed. It is suggested that early diagnosis, early surgery and combination of other treatment modalities should improve the outcome. This can only be possible with further training of health personnel including urologists, the education of the public and availability of improved diagnostic as well as treatment facilities especially cystoscopes and resectoscopes. There is need for developing proper management protocols for bladder tumours.

\section{REFERENCES}

1. Varkarakis M.J., Gaeta J., More R.H., et al. Superficial bladder tumour: Aspects of clinical progression. Urol. 1974; 4: 414.

2. Orihula O., et al. The practical use of tumour marker determination in bladder washing specimens. Cancer. 1987; 60: 1009-1016.

3. Sarosdy M., et al. Results of a multicenter trial using the BTA test to monitor for and diagnose recurrent bladder cancer. J. Urol. 1995; 154: 379-384.
4. D'Hallewin M. and Baert L. Initial evaluation of the bladder tumour antigen test in superficial bladder cancer. J. Urol. 1996; 155: 475-476.

5. Soloway M.S., et al. Use of a new tumour marker, urinary NMP22 in the detection of occult or rapidly recurring transitional cell carcinoma of the urinary tract following surgical treatment. J. Urol. 1996; 156: 363-367.

6. Carter H.B., Amberson J.B., Bander N.H., et al. Newer diagnostic techniques for bladder cancer. Urol. Clin. North Amer. 1987; 14: 763.

7. Oosterlink N., Kurth K.H., Schroder F., Bultinck J., Hamnmond B. and Sylivester R. A prospective EORTC trial comparing transurethral resection followed by single dose epirubicin or water in single stage $\mathrm{Ta}, \mathrm{T} 1$ papillary carcinoma of the bladder. J. Urol. 1993; 149: 749-752.

8. Lamm P.I., Blumenstein B.A., Crawford E.D., et al. A randomised trial of intravesical doxorubicin and immunotherapy with BCG for transitional cell of the urinary bladder. N. Engl. J. Med. 1991; 325: 1205-1209.

9. Vegt P.D.J., Witjes A., Witjes W.P.J., et al. A randomised study of intravesical mitomycin C, BCG Tice and BCG Rium treatment of pTa-pT1 papillary carcinoma and carcinoma in situ of the bladder. J. Urol. 1995; 153: 929-933.

10. Raghavan D. Review of pre-emptive (neoadjuvant) intravenous chemotherapy for invasive bladder carcinoma. Brit. J. Urol. 1998; 81: 1-6.

11. Martinez-Pineiro J.A. and Martinez-Pineiro L. The role of neoadjuvant chemotherapy for invasive bladder cancer. Brit. J. Urol. 1998; 82: 33-42.

12. Oldbring J., Glifberg J., Mikuloski P., et al. Carcinoma of the renal pelvis and ureter following bladder carcinoma: Frequency, risk factors and clinicopathological findings. J. Urol. 1989; 141: 1311.

13. Weldon T.E. and Soloway M.S. Susceptibility of urothelium to neoplastic cellular implantation. Urol. 1975; 5: 824.

14. Van der Werf-Messing B.H.P. Carcinoma of the urinary bladder treated by interstitial radiotherapy. Urol. Clin. North Amer. 1984; 11: 659.

15. William J. C. Urothelial tumours of the urinary tract. Campbells Urol. 1992; 2: 1094-1133.

16. Waihenya C.G. and Mungai P.N. Pattern of transitional cell carcinoma of the urinary bladder as seen in Kenyatta National Hospital. East Afr. Med. J. 2004; 81: $114-119$. 